\title{
Thermogravimetric analysis during the decomposition of cotton fabrics in an inert and air environment
}

\author{
Julia Moltó*, Rafael Font, Juan A. Conesa, Ignacio Martín-Gullón \\ Chemical Engineering Department, University of Alicante, P.O. Box 99, E-03080 Alicante, Spain \\ Received 29 November 2004; accepted 4 September 2005
}

\begin{abstract}
The thermal degradation of samples of used cotton fabrics has been investigated using thermogravimetric analysis (TGA) between room temperature and $700{ }^{\circ} \mathrm{C}$. Experiments were carried out with about $5 \mathrm{mg}$ of sample in three different atmospheres: helium, 20\% oxygen in helium and $10 \%$ oxygen in helium. Three different heating rates were used at each atmosphere condition. A kinetic model for the decomposition of used cotton fabrics explaining the behavior of all the runs performed has been proposed and tested. For the pyrolysis of the cotton, the model comprises two parallel reactions. For the combustion process, one competitive reaction was added to each parallel reaction of the pyrolysis model and four combustion reactions of the different solid fractions to obtain volatiles. One single set of parameters can explain all the experiments (pyrolysis, oxidative pyrolysis and combustion) at the three different heating rates used.
\end{abstract}

(C) 2005 Published by Elsevier B.V.

Keywords: Pyrolysis; Combustion; Cotton fabrics; Kinetics; Thermogravimetry

\section{Introduction}

The growing interest in renewable energies is accompanied by intensified research and development of technical processes for the thermal conversion of biomass and wastes. Used cotton fabrics could be used as biomass, and in this way, offer a valid alternative to disposal in landfills. Although cotton fabrics are usually recycled in other ways, thermal decomposition only of the wastes is interesting for some industries that mainly focus on obtaining the potential energy by combustion.

The development of pyrolysis-combustion processes requires an optimization of the operating conditions in order to assure both acceptable gas outlet composition and an energy recovery, which makes the process economically satisfactory [1]. A good knowledge of the kinetics of the process is fundamental for the plant design and scale-up bases on process simulation.

Cotton is mainly comprised of cellulose. The pyrolytic degradation of cellulose has been the subject of extensive study, although in many instances, knowledge of the exact nature of

\footnotetext{
* Corresponding author. Tel.: +34 9659034 00x3003; fax: +34 965903826. E-mail address: julia.molto@ua.es (J. Moltó).
}

degradation and decomposition remains incomplete. Chatterjee 43 and Conrad [2] studied the kinetics of cellulose decomposition 44 in the temperature range of $270-310{ }^{\circ} \mathrm{C}$ with absorbent cotton, 45 and proposed two series reactions for the pyrolysis process. 46 Dollimore and Hoath [3] used differential thermal analysis 47 (DTA) to follow the degradation of cellulose in air products and 48 obtained two and sometimes three exothermic peaks. Antal and 49 Várhegyi [4] reviewed the literature of cellulose pyrolysis and 50 concluded that the pyrolysis of a small sample of pure cellulose 51 is characterized by an endothermic reaction governed by a first 52 order rate law with a high activation energy. Völker and 53 Rieckmann [5] investigated the influence of the final mass on 54 modelling results and evaluated the applicability of established 55 kinetic models for engineering purposes.

Cotton fabrics, which have a major share of the textile 57 market, are highly inflammable and the development of 58 successful flame retardant systems for cotton is of major 59 interest. For this reason, many authors have studied the 60 mechanism of pyrolysis of untreated and flame retardant treated 61 cotton fabrics. For instance, Faroq et al. [6] carried out the 62 thermogravimetric analysis of the mechanism of pyrolysis of 63 untreated cotton fabrics and cotton fabrics finished with various 64 flame retardant, considering the fraction decomposed as 65 between 0.1 and 0.9 . These authors evaluated the activation 66 
66

67 energies obtaining values of $155-158 \mathrm{~kJ} \mathrm{~mol}^{-1}$ for the thermal 68 decomposition of untreated cotton. In addition, they proposed a 69 second order decomposition mechanism as the most suitable. 70 Wanna and Powell [7] studied the thermal decomposition of 71 untreated and treated cotton fabric with selected salts in 72 oxidative and inert atmospheres using TGA-FTIR. On the other 73 hand, no papers considering the overall decomposition kinetics 74 of cotton cellulose or cellulose have been found.

75 It is clear that the $n$-th order kinetic model can be correct for 76 homogeneous gas phase kinetics, in accordance with the 77 collision theory and the transition state theory developed for 78 elemental reactions and normally with $n=1,2$ or 3 . The 79 pyrolysis of polymers it is a non-homogeneous reaction and 80 consequently the $n$-th model should not be valid. In literature, 81 we can find mechanisms for the pyrolysis of different polymers 82 with parallel and series reactions. Considering only one 83 elemental reaction for the decomposition of a solid, the 84 proposal of a kinetic expression based on the elemental reaction 85 is not easy because the decomposition takes place in a solid 86 phase. Font and García [8] proposed the application of the 87 transition state theory to the pyrolysis of biomass considering 88 the similarity between the pyrolitic reaction that take places in 89 the outer surface and the first order uni-molecular catalytic 90 surface reactions. In accordance with the model developed, it is 91 possible to obtain $n$-th models as a consequence of the increase 92 or decrease of the surface with active centres where the 93 decomposition can take place, and with fractional values of the 94 reaction order $n$.

95 The $n$-th order reaction has been used extensively by 96 different researchers when studying the mechanisms of 97 decomposition of several polymers, no matter the mechanisms 98 of reactions comprises parallel or series reactions. Never99 theless, in spite of the proposal of a model that could explain 100 fractional reaction orders, the models obtained from the TG 101 runs must be considered as correlation ones, and from the 102 analysis of the activation energy, it can be deduced if the model 103 is related satisfactorily to a controlling decomposition 104 elemental step or must be only considered valid for correlation. 105 The present work, which is included in a wider project 106 whose objective is to study the combustion of different 107 industrial and municipal wastes, studies the thermal decom108 position of used and waste cotton fabrics from the thermo109 gravimetric point of view, including the kinetic analysis in a 110 thermobalance in inert atmosphere and with different amounts 111 of oxygen, proposing a kinetic model.

\section{Experimental}

112

\subsection{Raw material}

113

114 Used cotton fabrics were simulated by using a used blue T115 shirt made of $100 \%$ cotton. Prior to the runs, the T-shirt was cut 116 into small pieces with an average size of $1 \mathrm{~cm} \times 1 \mathrm{~cm}$.

117 Table 1 shows some characteristics of the material studied. 118 Elemental analysis of the major components was carried out in 119 a Perkin-Elmer 2400. The moisture was determined by the 120 weight loss at $105^{\circ} \mathrm{C}$ for $12 \mathrm{~h}$. The calorific value was obtained
Table 1

Characteristics of the material used

\begin{tabular}{ll}
\hline $\mathrm{C}$ (wt.\%) & 45.5 \\
$\mathrm{H}($ wt.\%) & 6.6 \\
$\mathrm{~N}($ wt.\%) & 0.3 \\
$\mathrm{~S}$ (wt.\%) & $<0.1$ \\
$\mathrm{O} \%$ by difference (wt.\%) & 47.5 \\
$\mathrm{Cl}$ (wt.\%) & 0.12 \\
Ash content (wt.\%) & $<0.1$ \\
Net calorific value (kJ kg & -1 \\
Moisture (wt.\%) & 17100 \\
\hline
\end{tabular}

in an AC-350 calorimetric bomb from Leco Corporation. Chlorine was measured using an automatic sequential spectrometer X-ray Fluorescence model TW 1480. Ash residue was obtained by calcination at $850{ }^{\circ} \mathrm{C}$.

\subsection{Thermobalance}

The thermogravimetric experiments were carried out in a Setaram thermobalance model DSC92 controlled by a PC system. The atmosphere used for pyrolysis was helium with a flow rate of $60 \mathrm{ml} \mathrm{min}^{-1}$ (STP), according to the specifications of the equipment. In the combustion runs, two mixtures of helium and oxygen: 9:1 (10\% oxygen) and 4:1 (20\% oxygen) were used, with the same total flow rate. The sample temperature was measured with a thermocouple directly at the crucible, i.e., very close to the sample. Because a water-cooled microfurnace was used, the temperature could be lowered rapidly.

Before the runs with used cotton fabrics, an experiment with a heating rate of $5{ }^{\circ} \mathrm{C} \mathrm{min}^{-1}$ using Avicel PH-105 microcrystalline cellulose was done to check the good performance of the equipment. The results obtained showed good agreement with the kinetic evaluation of Avicel Cellulose TG curves at this heating rate presented by Grønli et al. [9] in their round-robin study of cellulose pyrolysis kinetics by thermogravimetry.

The experiments were carried out with heating rates of 5, 10 and $20{ }^{\circ} \mathrm{C} \mathrm{min}{ }^{-1}$ over a variety of temperatures that included the entire range of solid decomposition, $80-700{ }^{\circ} \mathrm{C}$. Experiments without a sample were carried out, and used as background in order to subtract the buoyancy effect. The mass of the samples used was approximately $5 \mathrm{mg}$, and under these conditions the heat transfer limitations can be neglected.

\section{Results and discussion}

\subsection{Thermogravimetric study}

Figs. 1-3 show in detail the experimental curves for used cotton fabrics pyrolysis (helium) and combustion (helium:oxygen, 4:1 and 9:1) at different heating rates. The calculated curves of the kinetic models are also shown. In the figures, $w$ is defined as the residual mass fraction of the solid (including residue formed and non-reacted initial solid), i.e., the ratio between the solid mass at any time $(m)$ and the initial solid mass $\left(m_{0}\right)$. In all the processes, we can be observed the general shift to higher temperatures when the heating rate is increased.

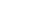

(1)

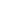

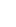

5

6

7

0

1

3

4

5

7




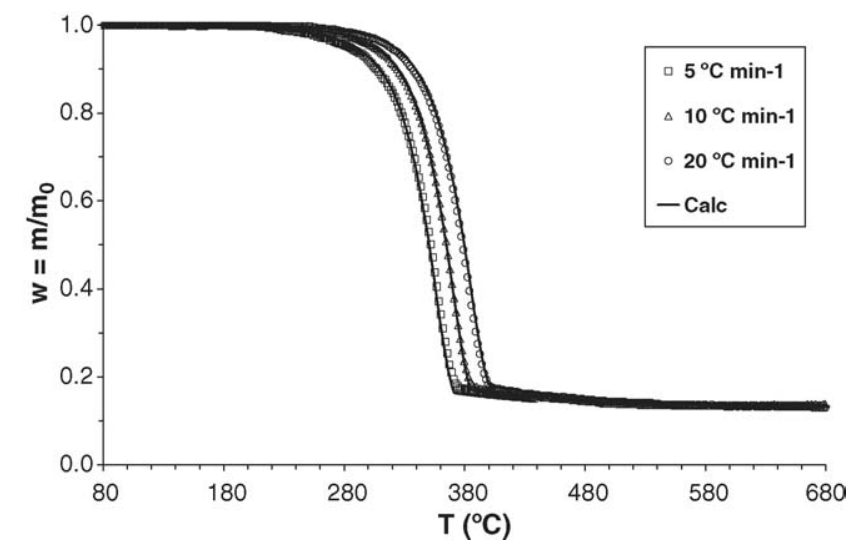

Fig. 1. Cotton pyrolysis at several heating rates: 5,10 and $20{ }^{\circ} \mathrm{C} \mathrm{min}^{-1}$. Experimental and calculated curves.

160
Fig. 4 compares the results obtained in the different atmospheres at a constant heating rate. As can be seen, the presence of oxygen accelerates the decomposition (taking place at lower temperatures) and a final combustion process is observed. On the other hand, there is not a great difference between the runs performed under $10 \%$ oxygen and those at $20 \%$ oxygen.

\subsection{Kinetics of the process}

\subsubsection{Pyrolysis model}

Most of the papers published corresponding to the pyrolysis of cellulose and cotton consider only one fraction when correlating the experimental data of the primary decomposition, in spite of the parallel and series mechanisms proposed in literature [4]. In the cotton fabrics used in this work, and considering that we have extended the temperature range until high temperatures, a second decomposition process has been considered in order to improve the correlation.

The kinetic model proposed for the decomposition of the used cotton fabrics in an inert atmosphere could be interpreted

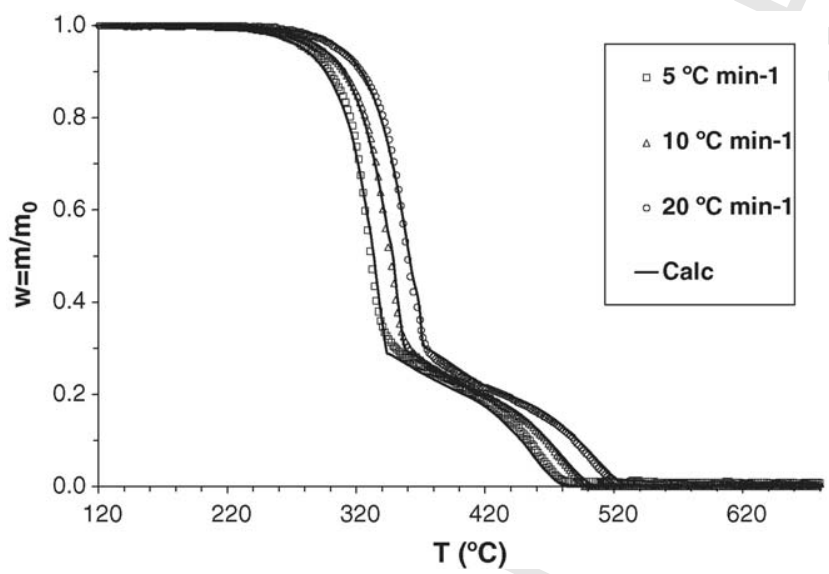

Fig. 2. Cotton combustion at several heating rates and 20\% oxygen. Experimental and calculated curves.

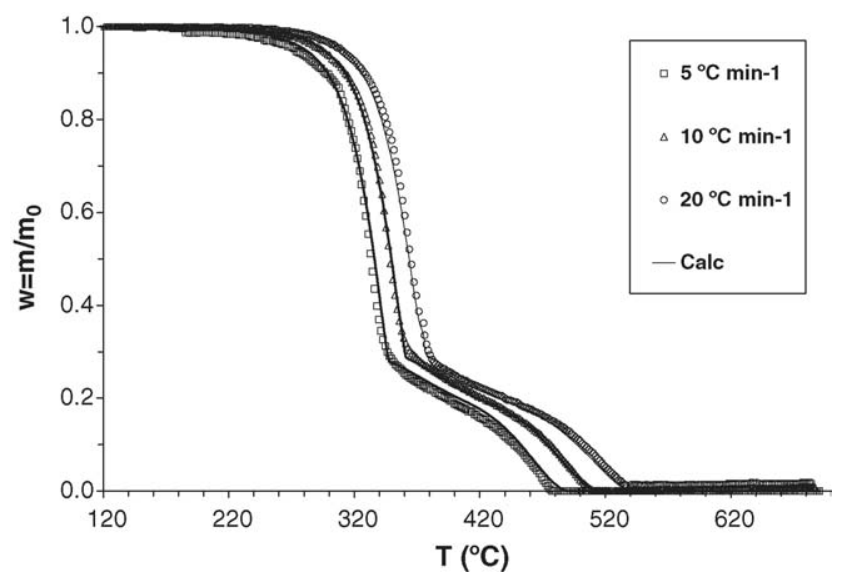

Fig. 3. Cotton combustion at several heating rates and 10\% oxygen. Experimental and calculated curves.

considering this waste formed by two independent parts, each 181 one following an independent reaction, as follows:

$c_{1} C_{1} \stackrel{1}{\longrightarrow} v_{1} V_{1}+s_{1} S_{1}$

$c_{2} C_{2} \stackrel{2}{\longrightarrow} v_{2} V_{2}+s_{2} S_{2}$

where $C_{1}$ and $C_{2}$ refer to different parts of the solid material to be decomposed (cotton in this case). " $V_{i}$ " are the gases + volatiles evolved and " $S_{i}$ " are the solid residue formed in the decomposition. The uncapitalized variables " $c_{n}$ " are the amount of each material that is in the sample.

It is very useful to introduce the concept of the conversion degree for each reaction:

$\alpha_{i}=1-\frac{C_{n}}{C_{n \mathrm{o}}}=\frac{V_{i}}{V_{i \infty}}, \quad i=n=1,2$

(Two different subscripts have been used, because in the 195 combustion model presented also in this model there are 196 competitive reactions for the same solid.)

In the previous equations, $V_{i \infty}$ represents the maximum 198 obtainable amount of volatiles via reaction ' $i$ ' at time infinity,

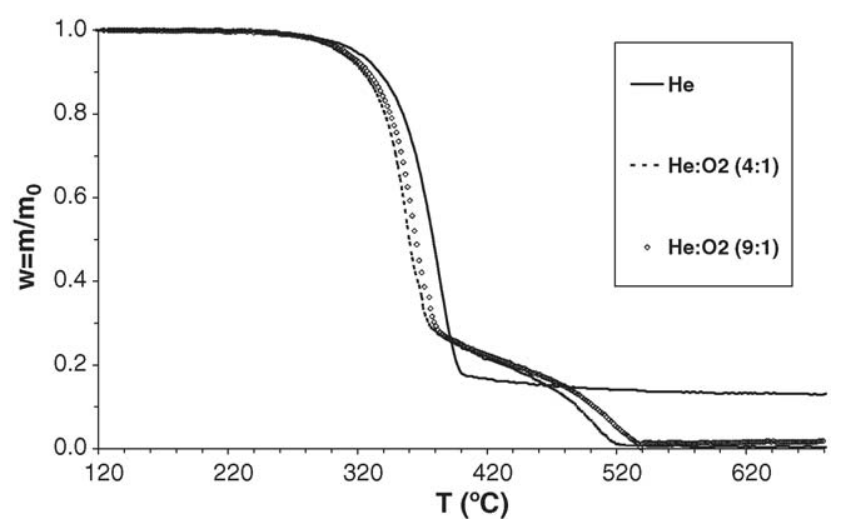

Fig. 4. Experimental TG plots for pyrolysis and combustion with 20 and $10 \%$ oxygen, all carried out at $20^{\circ} \mathrm{C} \mathrm{min}-1$ heating rate. 
$199 C_{n}$ refers to the non-decomposed material at each time, and $C_{n \text { o }}$ 200 is the initial contribution of fraction ' $n$ ' to the total weight. Note 201 that at $t=0$ the value of $\alpha_{i}$ is zero, and that $V_{1 \infty}$ equals the yield 202 coefficient $v_{1}$ and that $V_{2 \infty}$ equals the yield coefficient $v_{2}$.

203 The kinetic equations associated with the parallel reaction 204 for the pyrolysis runs, taking into account the mass balance 205 between products and reactants and the degree conversions, can 206 be expressed as:

$$
\left.\begin{array}{l}
-\frac{\mathrm{d}\left(\frac{C_{n}}{C_{n \mathrm{o}}}\right)}{\mathrm{d} t}=\frac{\mathrm{d}\left(\frac{V_{i}}{V_{i \infty}}\right)}{\mathrm{d} t}=\frac{\mathrm{d} \alpha_{i}}{\mathrm{~d} t}=k_{i}\left(\frac{C_{n}}{C_{n \mathrm{o}}}\right)^{n_{i}}=k_{i}\left(1-\alpha_{i}\right)^{n_{i}} \\
k_{i}=k_{i \mathrm{o}} \exp \left(-\frac{E_{i}}{R T}\right)
\end{array}\right\}, \quad n, i=1,2
$$

By integration of these reactions it is possible to calculate $\alpha_{1}$ and $\alpha_{2}$ at each time; the relationship between these two values and the weight fraction measured in the thermobalance $(w)$ is:

212 The values of $V_{1 \infty}$ and $V_{2 \infty}$ are related with the total volatiles at time infinity $\left(V_{\infty}\right)$, that is a known amount:

${ }_{213} V_{\infty}=V_{1 \infty}+V_{2 \infty}$

216 The pyrolysis data and the combustion data have been 217 correlated together, in order to obtain a single set of parameters 218 for the thermal decomposition of used cotton fabrics in different 219 atmospheres: pyrolysis, oxidative pyrolysis and combustion.

\section{3.2.2. Combustion model}

221 Similarly to the kinetic models found in literature to explain 222 pyrolytic processes, different authors propose different models 223 to explain decomposition mechanisms under oxidative atmo224 spheres. Obviously, if the model proposed satisfactorily fits 225 experimental data generated under a wide selection of 226 conditions, the model can be considered representative of 227 the process analyzed [10]. However, literature that includes 228 detailed kinetic studies, fitting experimental curves at different 229 heating rates and oxygen content is extremely sparse. Different

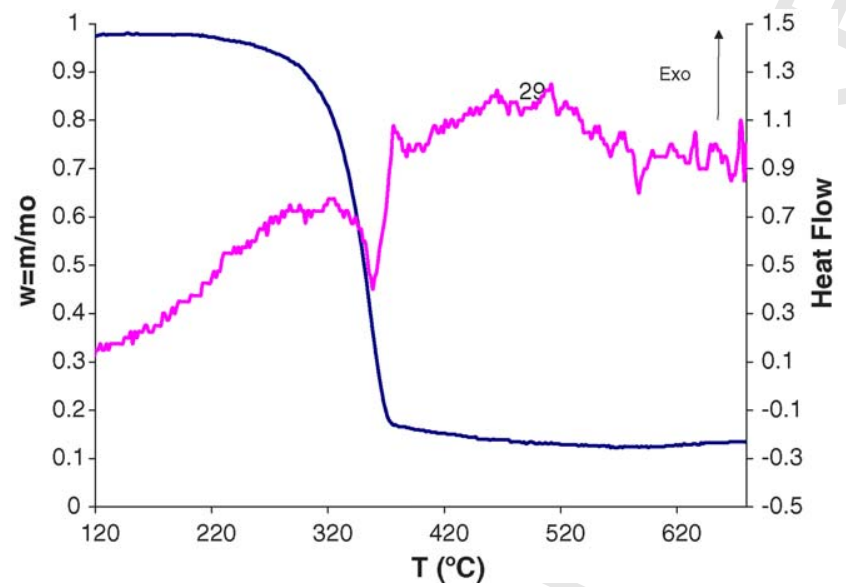

Fig. 5. Experimental TG and DSC plots for pyrolysis at $5{ }^{\circ} \mathrm{C} \min ^{-1}$ heating rate. models have been found in literature, concerning the decomposition of tannery waste under oxygenated atmosphere [11], polycoated materials such as milk cartons [12], polytetrafluoroethylene [13] and also of tire wastes [14]. No models for combustion of cotton or used cotton fabrics have been found, as commented previously.

The kinetic model proposed for the combustion runs could be interpreted considering, that the presence of the oxygen introduces a new competitive process for the decomposition of each fraction and in this way this model explains the fact observed in Fig. 4, where the first weight loss in combustion runs reaches a lower value than in pyrolysis runs.

Figs. 5 and 6 show the TG and DSC plots for pyrolysis and combustion at $5{ }^{\circ} \mathrm{C} \min ^{-1}$ heating rate. As seen in Fig. 5 , an endothermic peak appears for the pyrolysis process and in the combustion process besides two exothermic peaks appear.

Taking into account the behavior commented above, the oxygen to be included in the decomposition law has been considered, as has been done with other materials [15-16].

The following scheme represents the combustion model proposed to explain the behaviour obtained at two different helium: oxygen atmospheres and three heating rates:

$$
\begin{aligned}
& c_{1} C_{1} \stackrel{1}{\longrightarrow} v_{1} V_{1}+s_{1} S_{1} \\
& c_{1} C_{1}+O_{2} \stackrel{3}{\longrightarrow} v_{3} V_{3}+s_{3} S_{3} \\
& c_{2} C_{2} \stackrel{2}{\longrightarrow} v_{2} V_{2}+s_{2} S_{2} \\
& c_{2} C_{2}+O_{2} \stackrel{4}{\longrightarrow} v_{4} V_{4}+s_{4} S_{4}
\end{aligned}
$$

237

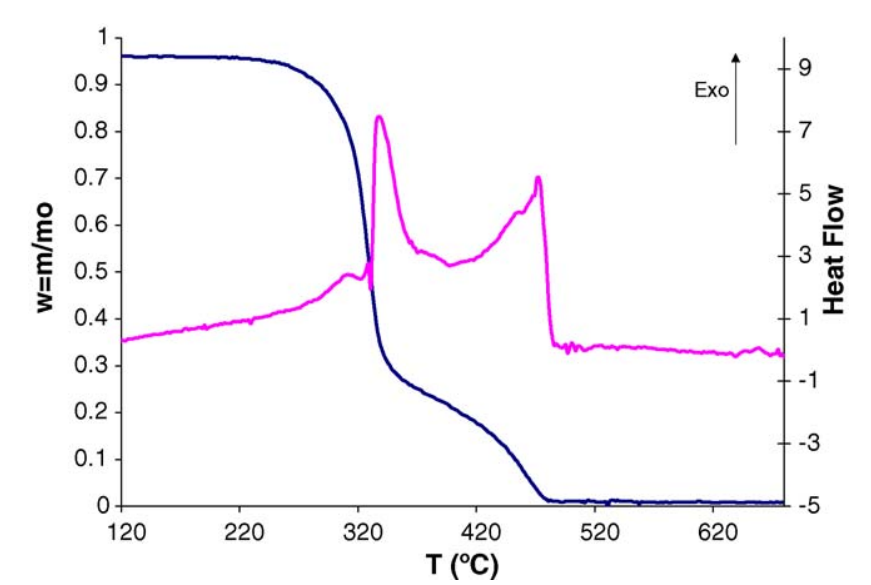

Fig. 6. Experimental TG and DSC plots for combustion with $20 \%$ oxygen at $5^{\circ} \mathrm{C} \min ^{-1}$ heating rate. 
Reactions 1 and 2 are the same as in pyrolysis. Furthermore, as a final new process appears in combustion runs, the combustion reactions to the chars formed has been considered:

$s_{i} S_{i}+O_{2} \stackrel{5}{\longrightarrow} s_{i} V_{\mathrm{c} i}, \quad i=1,2,3,4$

$V_{\mathrm{c} i}$ refers to the volatiles produced through the combustion reaction with solid $S_{i}$.

Conversion degrees are considered for each reaction:

$\alpha_{i}=1-\frac{\left(C_{n}\right)_{\text {reacted by reaction } i}}{C_{n \mathrm{o}}}=\frac{V_{i}}{V_{i \infty}}$,

$i=1,2,3,4 ; n=1($ for $i=1,3) ; n=2($ for $i=2,4)$

$\alpha_{\mathrm{c} i}=\frac{\left(S_{i}\right)_{\text {burnt }}}{s_{i}}=\frac{V_{\mathrm{c} i}}{V_{\mathrm{c} i \infty}}$,

$i=1,2,3,4 ; n=1($ for $i=1,3) ; n=2($ for $i=2,4)$

where $V_{i \infty}$ and $V_{\text {ci } \infty}$ represent the maximum amounts of volatiles evolved if the whole solid fraction decomposes only through the reactions that lead to the corresponding volatiles, without competitive reaction. Note that in this case $V_{i \infty}$ equals the yield coefficient $v_{i}$ and that $V_{\text {cio }}$ equals the yield coefficient $s_{i}$.

Reactions 1 and 3 are competitive with respect to the same solid $C_{1}$, so it is possible that none of the two values of degree conversion $\alpha_{1}$ and $\alpha_{2}$ can reach the value 1 at time infinity, although the sum $\alpha_{1}$ and $\alpha_{2}$ logically must be equal to 1 , when the reactant $C_{1}$ is exhausted. In this case, the ratio between the non-reacted solid $C_{1}$ and the initial solid $C_{10}$, taking into account the degree conversions $\alpha_{1}$ and $\alpha_{3}$, can be expressed as:

$\frac{C_{1}}{C_{10}}=1-\alpha_{1}-\alpha_{3}$

and consequently the kinetic laws for the decomposition of the solid fraction $C_{1}$ can be written as

$-\frac{\mathrm{d}\left(\frac{C_{1}}{C_{1 \mathrm{o}}}\right)_{\text {by reaction } i}}{\mathrm{~d} t}=\frac{\mathrm{d} \alpha_{i}}{\mathrm{~d} t}=k_{i}\left(\frac{C_{1}}{C_{1 \mathrm{o}}}\right)=k_{i}\left(1-\alpha_{1}-\alpha_{3}\right)^{n_{i}}$,

$$
i=1,3
$$

Similarly for reactions 2 and 4 , the following expression can be obtained:

$$
-\frac{\mathrm{d}\left(\frac{C_{2}}{C_{20}}\right)_{\text {by reaction } i}}{\mathrm{~d} t}=\frac{\mathrm{d} \alpha_{i}}{\mathrm{~d} t}=k_{i}\left(\frac{C_{2}}{C_{2 \mathrm{o}}}\right)=k_{i}\left(1-\alpha_{2}-\alpha_{4}\right)^{n_{i}},
$$

$i=2,4$

For the combustion of the residue $S_{1}$ (formed by the first reaction) in accordance with the scheme:

$s_{1} S_{1}+O_{2} \stackrel{5}{\longrightarrow} s_{1} V_{\mathrm{c} 1}$

The degree conversion $\alpha_{\mathrm{c} 1}$ is

$\alpha_{\mathrm{c} 1}=\frac{\left(S_{1}\right)_{\mathrm{burnt}}}{s_{1}}=\frac{V_{\mathrm{c} 1}}{V_{\mathrm{c} 1 \infty}}$
The kinetic law for the combustion of $S_{1}$ can be written as:

$-\frac{\mathrm{d}\left(S_{1} / s_{1}\right)_{\text {burnt }}}{\mathrm{d} t}=k_{5}\left(\frac{S_{1}}{s_{1}}\right)^{n_{5}}$

In this way, the kinetic constant of this second decomposi- 303 tion does not depend on the initial mass fraction, and 304 consequently the kinetic constant $k_{5}$ with distinct mass 305 fractions.

On the other hand, it can be deduced that:

$$
\left(\frac{S_{1}}{s_{1}}\right)_{\text {at any time }}=\left(\frac{S_{1}}{s_{1}}\right)_{\text {formed reaction 1 }}-\left(\frac{S_{1}}{s_{1}}\right)_{\text {burnt }}=\alpha_{1}-\alpha_{\mathrm{c} 1}
$$

Consequently, it can be written that:

$\frac{\mathrm{d} \alpha_{\mathrm{c} 1}}{\mathrm{~d} t}=k_{5}\left(\alpha_{1}-\alpha_{\mathrm{c} 1}\right)^{n_{5}}$

Another way of obtaining similar expressions can be found 313 elsewhere [17]. This procedure can be applied to the other three 314 combustion reactions. Consequently, the reaction model can be 315 solved considering the following equations:

$\frac{\mathrm{d} \alpha_{i}}{\mathrm{~d} t}=k_{i}\left(1-\alpha_{1}-\alpha_{3}\right)^{n_{i}}, \quad i=1,3$

$\frac{\mathrm{d} \alpha_{i}}{\mathrm{~d} t}=k_{i}\left(1-\alpha_{2}-\alpha_{4}\right)^{n_{i}}, \quad i=2,4$

$\frac{\mathrm{d} \alpha_{1 i}}{\mathrm{~d} t}=k_{5}\left(\alpha_{i}-\alpha_{1 i}\right)^{n_{5}}, \quad i=1,2,3,4$

$v_{1}=V_{1 \infty} ; \quad v_{2}=V_{2 \infty} ; \quad v_{3}=V_{3 \infty} ; \quad v_{4}=V_{4 \infty}$

$s_{1}=V_{\mathrm{c} 1 \infty} ; \quad s_{2}=V_{\mathrm{c} 2 \infty} ; \quad s_{3}=V_{\mathrm{c} 3 \infty} ; \quad s_{4}=V_{\mathrm{c} 4 \infty}$

$v_{1}+s_{1}=v_{3}+s_{3}$

$v_{2}+s_{2}=v_{4}+s_{4}$

Note that the same kinetic constant and reaction order are 332 considered for the decomposition of $S_{i}$.

The total weight fraction is related to the other variables by:

$$
\begin{aligned}
w & =1-V \\
& =1-\left(V_{1}+V_{2}+V_{3}+V_{4}+V_{11}+V_{12}+V_{13}+V_{14}\right)
\end{aligned}
$$

$w=1$

$$
\begin{aligned}
& -\left(\alpha_{1} V_{1 \infty}+\alpha_{2} V_{2 \infty}+\alpha_{3} V_{3 \infty}+\alpha_{4} V_{4} \infty+\alpha_{\mathrm{c} 1} V_{\mathrm{c} 1 \infty}\right. \\
& \left.+\alpha_{\mathrm{c} 2} V_{\mathrm{c} 2 \infty}+\alpha_{\mathrm{c} 3} V_{\mathrm{c} 3 \infty}+\alpha_{\mathrm{c} 4} V_{\mathrm{c} 4 \alpha}\right)
\end{aligned}
$$

To take into account the effect of the partial pressure of 339 oxygen (which equals 0.10 and $0.20 \mathrm{~atm}$ for helium:oxygen 9:1 340 and $4: 1$, respectively), since different behaviour is observed 341 when comparing corresponding thermograms, the pre-expo- 342 nential factors for reactions with oxygen have been considered 343 to consist of two terms, one a typical pre-exponential factor $k_{i 0}^{\prime} \quad 344$ and the other one the partial pressure of oxygen $P_{\mathrm{O}_{2}}$ raised to the power of an order $b_{i \mathrm{o}}$ :

$k_{i \mathrm{o}}=k_{i \mathrm{o}}^{\prime}\left(P_{\mathrm{O}_{2}}\right)^{b_{i}}, \quad i=3,4,5$ 
348

The parameters optimized were: five orders of reaction, five 349 pre-exponential factors, five activation energies and $v_{1}, v_{2}, v_{3}$, $350 v_{4}, s_{1}, s_{2}$, and the values of $b_{3}, b_{4}, b_{5}$. A simplification made in 351 the model is to assume the same dependency of the constants 352 with the partial pressure of oxygen, in the combustion of the 353 chars.

354 The objective function (OF) to minimize was the sum of the 355 square differences between experimental and calculated weight loss values $\mathrm{OF}=\sum_{m=1}^{3} \sum_{j=1}^{N}\left(w_{m j}^{\exp }-w_{m j}^{\text {calc }}\right)^{2}, \quad m$, heating rates; $j$, points

359 The model validity has been tested calculating the variation coefficient (VC):

$$
360 \mathrm{VC}=\frac{\sqrt{\mathrm{OF} /(N-P)}}{\overline{w_{\exp }}} \times 100
$$

362 where $N$ and $P$ are the number of data and parameters fitted, 363 respectively, and $\overline{w_{\exp }}$ is the average of the experimental 364 weights. According to the procedure suggested by Martín365 Gullón et al. [18], the great interrelation existing among the 366 pre-exponential factor, the apparent activation energy and the 367 reaction order can be decreased when optimization is per368 formed in terms of a 'comparable kinetic constant' $K_{i}^{*}$ instead 369 of optimizing $k_{\mathrm{o} i}$. This constant is calculated in a temperature 370 around the maximum decomposition rate $\left(T_{\max }\right)$. Since $K_{i}^{*}, E_{i}$ 371 and $n_{i}$ are optimised the pre-exponential factor $k_{\mathrm{o} i}$ is calculated using the following expression:

$K_{i}^{*}=k_{i}(0.64)^{n_{i}}=k_{\mathrm{o} i} \exp \left(\frac{-E_{i}}{R T_{\max }}\right)(0.64)^{n_{i}}$

Conesa et al. $[19,20]$ came to a conclusion in their study of TG-DTG curves that at least three TG curves with different heating rates must be adjusted simultaneously in order for a kinetic model to be considered as potentially correct.

The kinetic parameters have been optimized in order to minimize the differences between experimental and calculated weight loss. Table 2 presents the values of the optimised parameters. Note that all the data of the three atmospheres 385 As can be seen in Figs. 1-4, the model is able to explain 386 all the experimental data collected, at all the heating rates 387 studied and in the presence and absence of oxygen. Bear in 388 mind that all the runs are fitted with no variation of the 389 parameters.

390 On analysing the values of the parameters in Table 2, some interesting conclusions can be obtained:

393 1. Considering the pyrolysis parameters, it can be observed that fraction 1 has a value of $v_{1}$, that equals a $V_{1 \infty}$, around 0.75 , indicating that this fraction is the most important, although there is a small but significant second fraction, with a value $v_{2}$ around 0.11. Analysing the TG data of some papers concerning the pyrolysis of cellulose, this second fraction is also present [21,5].
Table 2

Kinetic parameters obtained for the thermal decomposition of used cotton fabrics $\left(k_{i \mathrm{o}}^{\prime}\right.$ in $\mathrm{s}^{-1} \mathrm{~atm}^{-b_{i}}, E_{i}$ in $\left.\mathrm{kJ} \mathrm{mol}^{-1}\right)$

\begin{tabular}{|c|c|}
\hline \multicolumn{2}{|l|}{ Pyrolysis } \\
\hline$k_{10}^{\prime}$ & $9.76 \times 10^{10}$ \\
\hline$E_{1}$ & 161.1 \\
\hline$n_{1}$ & 0.57 \\
\hline$v_{1}$ & 0.749 \\
\hline$s_{1}$ & 0.103 \\
\hline$k_{2 \mathrm{o}}^{\prime}$ & $1.32 \times 10^{3}$ \\
\hline$E_{2}$ & 65.2 \\
\hline$n_{2}$ & 2.62 \\
\hline$v_{2}$ & 0.124 \\
\hline$s_{2}$ & 0.024 \\
\hline \multicolumn{2}{|c|}{ Oxidative pyrolysis } \\
\hline$k_{30}^{\prime}$ & $2.70 \times 10^{11}$ \\
\hline$b_{3}$ & 0.39 \\
\hline$E_{3}$ & 159.4 \\
\hline$n_{3}$ & 0.29 \\
\hline$v_{3}$ & 0.589 \\
\hline$s_{3}$ & 0.263 \\
\hline$k_{4 \mathrm{o}}^{\prime}$ & $1.33 \times 10^{23}$ \\
\hline$b_{4}$ & 0.57 \\
\hline$E_{4}$ & 306.4 \\
\hline$n_{4}$ & 1.49 \\
\hline$v_{4}$ & 0.148 \\
\hline$s_{4}$ & 0 \\
\hline \multicolumn{2}{|c|}{ Combustion of char } \\
\hline$k_{50}^{\prime}$ & $4.47 \times 10^{6}$ \\
\hline$b_{5}$ & 0.31 \\
\hline$E_{5}$ & 126.8 \\
\hline & 0.64 \\
\hline $\mathrm{VC}(\%)$ & 2.628 \\
\hline
\end{tabular}

2. Concerning the kinetic parameters of the main fraction in the pyrolysis decomposition, activation energy around $161 \mathrm{~kJ} \mathrm{~mol}^{-1}$ and reaction order around 0.57 are obtained, that are similar to the values proposed by Antal et al. (1980) when considering all the runs. The activation energy $E_{1}$ obtained is also very similar to the values calculated by Faroq et al. [6] using two different methods: the iso-conversional technique and the most probable mechanism.

3. With respect to the minor fraction in pyrolysis, a low value of activation energy around $65 \mathrm{~kJ} \mathrm{~mol}^{-1}$ and a reaction order equal to 2.77 are obtained, as a consequence of the slow decomposition of this fraction at high temperatures, and consequently these values can be considered as correlation parameters with no physical meaning.

4. The oxidative pyrolysis of the main fraction has activation energy $\left(159 \mathrm{~kJ} \mathrm{~mol}^{-1}\right)$ similar to that of pyrolysis $\left(161 \mathrm{~kJ} \mathrm{~mol}^{-1}\right)$, and the reaction order $(0.29)$ is somewhat lower than in pyrolysis (0.57). An order reaction less than unity can be due to an increase of the active surface with the reaction extension [8], so it is possible that in the oxidative process, the active surface increases more intensively than in pyrolysis. The value of $v_{3}(0.569)$, that equals $V_{3 \infty}$, is less than the value $\left(V_{1 \infty}=0.755\right)$ corresponding to the main fraction in pyrolysis. This fact could be explained as a 
consequence of the partial oxidation of the molecular organic chains to ketones, aldehydes, carboxylic acids, etc., causing a weight loss lesser than in the case without oxygen. Therefore, all these parameters can be considered as acceptable and logical from a physical-chemical point of view.

5. In the oxidative pyrolysis of the minor fraction, the activation energy is around $150 \mathrm{~kJ} \mathrm{~mol}^{-1}$, the reaction order is 0.97 and there is no formation of residue solid. These parameters must only be accepted for correlation of the data.

6. In the combustion of the chars, assuming the same kinetics for the three residue solids formed $\left(S_{1}, S_{2}\right.$ and $\left.S_{3}\right)$, the apparent activation energy is around $126 \mathrm{~kJ} \mathrm{~mol}^{-1}$. The apparent activation energy obtained for the combustion of the char formed $\left(126 \mathrm{~kJ} \mathrm{~mol}^{-1}\right)$ is similar to that obtained by other researchers. Haji-Sulaiman and Aroua [22] proposed values around $135 \mathrm{~kJ} \mathrm{~mol}^{-1}$ for the oxidation of Malaysian coal chars. Henrich et al. [23], also obtained values of apparent activation energy around $140 \mathrm{~kJ} \mathrm{~mol}^{-1}$ are for oxidation in pure oxygen of soot, graphite, activated charcoals and chars of municipal waste and electronic scrap. Nevertheless, Walker et al. [24] suggested apparent activation energy around $209-242 \mathrm{~kJ} \mathrm{~mol}^{-1}$ for the reaction: $\mathrm{C}+1 / 2 \mathrm{O}_{2} \rightarrow \mathrm{CO}$. A reaction order (0.57) less than unity is obtained. This latter aspect could also be explained as a consequence of the increase of the active surface with the reaction extension.

7. The reaction orders with respect to the oxygen for the oxidative pyrolysis are 0.39 for the main fraction and 0.45 for the smaller fraction, indicating a low dependence of this with respect to the oxygen. For the combustion of char the reaction order with respect to oxygen is 0.31 , that is a low value in comparison with respect to the reaction order between 0.7 and 1 considered in the gasification of the carbon [25]. The experimental data shown in Fig. 4 shows the small dependence of the oxygen on the TG runs. On the other hand, it is possible that the simplification of the model, considering that the three combustion reactions follow the same kinetic law causes the correlation parameters to be somewhat different to those expected.

A study of the main by-products formed in the pyrolysis and combustion of used cotton fabrics, was carried out in a very recent paper [26]. More than 90 compounds, including carbon oxides, light hydrocarbons and PAHs, have been identified and quantified. In the gas phase some of the main components obtained were methane, ethene and benzene. The main semivolatile compounds detected were styrene, phenol, naphthalene, acenaphthylene and phenanthrene.

The evolution of the different fractions throughout the heating process can be observed in Figs. 7 and 8, that show the experimental and calculated mass fraction of volatiles $v$ and conversion factors $\alpha$ at $5{ }^{\circ} \mathrm{C} \mathrm{min}{ }^{-1}$ in $\mathrm{He}: \mathrm{O}_{2} 4: 1$. The kinetics parameters for reaction 2 show that there is a second order reaction with a low activation energy, and this could explain that the decomposition of the fraction $\mathrm{C}_{2}$ occurs in a wide range of temperatures, as seen in the big separation between the curves of $\alpha_{2}$ and $\alpha_{4}$ (Fig. 8).

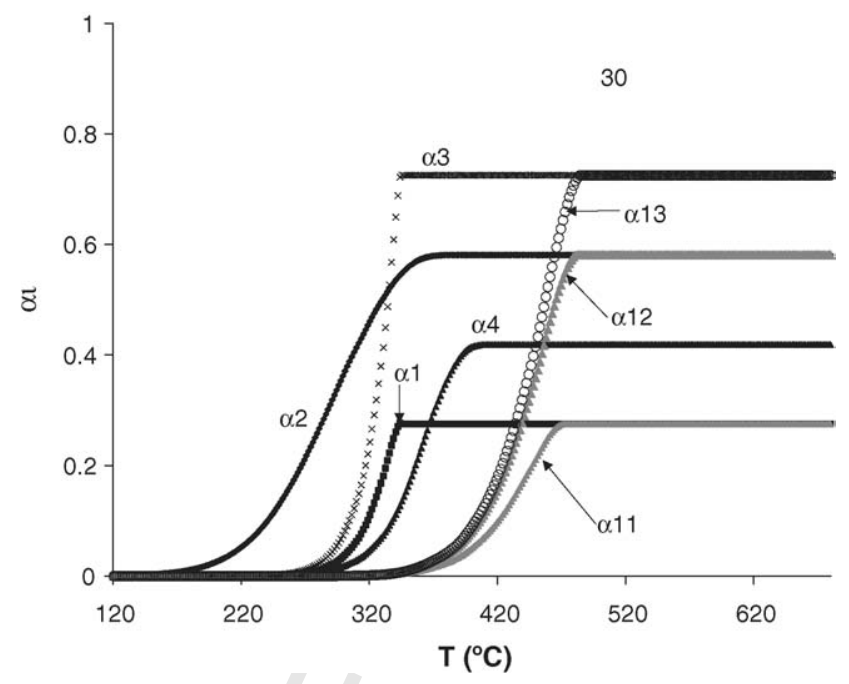

Fig. 7. Calculated conversion factor $\left(\alpha_{i}, \alpha_{i j}\right)$ at $5^{\circ} \mathrm{C} \min ^{-1}$ in $\mathrm{He}: \mathrm{O}_{2} 4: 1$.

It must be emphasised that the parameters obtained must be 488 considered as correlation ones, some of them with physical- 489 chemical significance. There is a great interrelation between 490 them, so other sets of parameters could be also valid for the 491 correlation of the data with a very small increase of the 492 objective function. In spite of this great interrelation of the 493 parameters, it must be indicated that the objective function has 494 been minimized reducing the great interrelation that exits 495 between the pre-exponential factor, the activation energy and 496 the reaction order.

497

The correlation of the three pyrolysis runs and six 498 combustion runs have been done simultaneously, so 26499 parameters were optimized for minimizing the objective 500 function. No papers have been found in literature where 501 pyrolysis and combustion runs were optimized simultaneously, 502 so the correlation work presented in this paper can be 503 considered as original. As a result, an acceptable set of 504 parameters is obtained, that correlates the nine TG runs 505

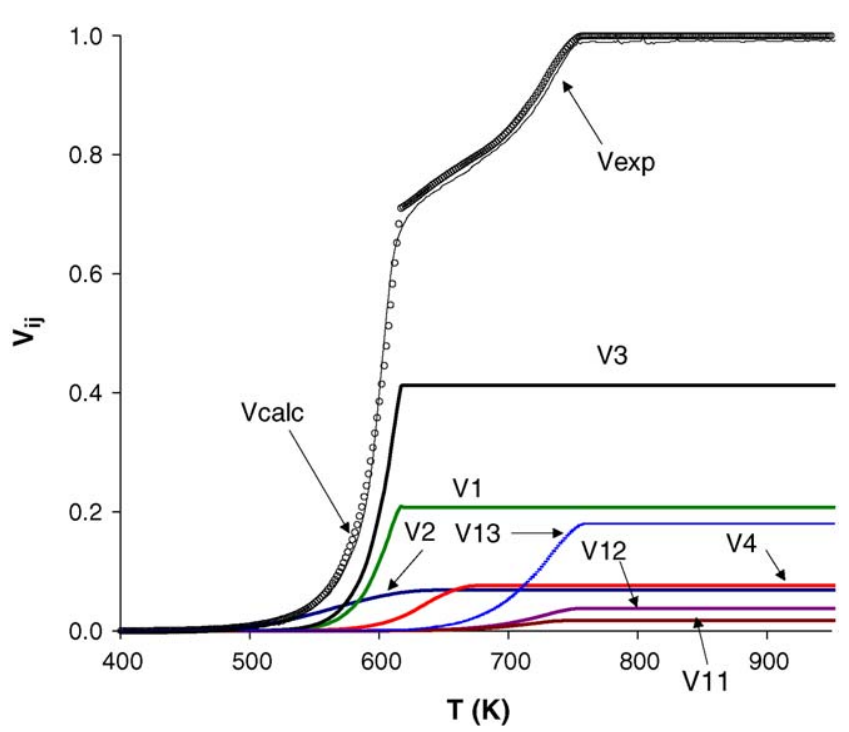

506

507

508

509

510

511

512

513

514

515

516

517

518

519

520

521

522

523

Fig. 8. Calculated mass fraction of volatiles $\left(v_{i j}\right)$ at $5{ }^{\circ} \mathrm{C} \mathrm{min}^{-1}$ in $\mathrm{He}: \mathrm{O}_{2} 4: 1.525$ 
526 satisfactorily, as can be seen in Figs. 1-3 that show the 527 conversion degrees versus temperature for a run, observing a 528 logical variation in accordance with the parameters deduced.

\section{Acknowledgments}

530 Support for this work was provided by the Ministerio de 531 Educación y Ciencia of Spain, research projects PPQ200253200567 and PPQ2002-10548-E.

\section{References}

533

534

535

536

537

538

539

540

541

542

543

544
[1] J.A. Caballero, R. Font, M.M. Esperanza, J. Anal. Appl. Pyrol. 47 (1998) 165.

[2] P.K. Chatterjee, C.M. Conrad, Text. Res. J. 36 (1966) 487.

[3] D. Dollimore, J.M. Hoath, Thermochim. Acta 45 (1981) 87.

[4] M.J. Antal, G. Várhegyi, Ind. Eng. Chem. Res. 34 (1995) 703.

[5] S. Völker, Th. Rieckmann, J. Anal. Appl. Pyrol. 62 (2002) 165.

[6] A.A. Faroq, D. Price, G.J. Milnes, Polym. Degrad. Stab. 44 (1994) 323.

[7] J.T. Wanna, J.E. Powell, Thermochim. Acta 226 (1993) 257.

[8] R. Font, A.N. García, J. Anal. Appl. Pyrol. 35 (1995) 249.

[9] M. Grønli, M.J. Antal, G. Várhegyi, Ind. Eng. Chem. Res. 38 (1999) 2238.

[10] J.A. Conesa, R. Font, A. Marcilla, J.A. Caballero, J. Anal. Appl. Pyrol. 5859 (2001) 619.
[11] J.A. Caballero, R. Font, M.M. Esperanza, J. Anal. Appl. Pyrol. 47 (1998) 165.

[12] J.A. Reyes, J.A. Conesa, A. Marcilla, J. Anal. Appl. Pyrol. 58-59 (2001) 747.

[13] J.A. Conesa, R. Font, Polym. Eng. Sci. 41 (2001) 2137.

[14] J.A. Conesa, R. Font, A. Fullana, J.A. Caballero, Fuel 77 (1998) 1469.

[15] S. Vossoughi, Y.M. El-Shoubary, Thermochim. Acta 157 (1995) 37.

[16] I. Martín-Gullón, M. Esperanza, R. Font, J. Anal. Appl. Pyrol. 58-59 (2001) 635.

[17] R. Font, I. Martín-Gullón, M. Esperanza, A. Fullana, J. Anal. Appl. Pyrol. 58-59 (2001) 703.

[18] I. Martín-Gullón, M.F. Gómez-Rico, A. Fullana, R. Font, J. Anal. Appl. Pyrol. 68-69 (2003) 645.

[19] J.A. Conesa, J.A. Caballero, A. Marcilla, R. Font, Thermochim. Acta 254 (1995) 175.

[20] J.A. Conesa, A. Marcilla, R. Font, J.A. Caballero, J. Anal. Appl. Pyrol. 36 (1996) 159.

[21] M.J. Antal, G. Várhegyi, E. Jakab, Ind. Eng. Chem. Res. 37 (1998) 1267.

[22] M.Z. Haji-Sulaiman, M.K. Aroua, J. Inst. Energy 70 (1997) 52.

[23] E. Henrich, S. Bürkle, Z.I. Meza-Renken, S. Rumpel, J. Anal. Appl. Pyrol. 9 (1999) 133.

[24] P.L. Walker, F. Rusinko, L.G. Austin, Adv. Catal. 9 (1959) 133.

[25] Z. Du, A.F. Sarofim, J.P. Longwell, in: J. Lahay, P. Ehrburger (Eds.), Fundamental Issues in Control of Carbon Gasification, 1992, p. 91.

[26] J. Moltó, J.A. Conesa, R. Font, I. Martín-Gullón, Environ. Sci. Technol. 39 (14) (2005) 5141. 